\title{
HYPOELLIPTICITY IN THE COMPLEXES OF PSEUDODIFFERENTIAL OPERATORS
}

\author{
JONGSIK KIM
}

\begin{abstract}
Sufficient conditions for the pseudodifferential operator defined on a complex to be hypoelliptic are investigated.
\end{abstract}

Introduction. In this paper we shall continue the study of hypoellipticity in the complexes of pseudodifferential operators introduced in Treves [3]. In [3], Treves characterized the necessary and sufficient condition of the hypoellipticity of the pseudodifferential operators in the complexes in the 0 dimensional level. We shall study the case when dimension $p$ (i.e., $p$ th step in the complex) is greater than 0 .

In the first section we derive a plausible definition of hypoellipticity in the complexes of pseudodifferential operators in the $p>0$ dimensional level. In the second section we give certain sufficient conditions for the pseudodifferential operators to be hypoelliptic, while in the third section we discuss the case when the pseudodifferential operators are nondegenerate.

1. Hypoellipticity in the complexes of pseudodifferential operators. Let $R^{\boldsymbol{\nu}}$ (resp. $R^{n}$ ) be a $\nu$-dimensional (resp. $n$-dimensional) Euclidean space. Throughout this paper, we shall denote by $\Omega$ a fixed open subset of $R^{\nu}$. For any real number $s$ we denote by $H^{s}=H^{s}\left(R^{n}\right)$ the standard global Sobolev space on $R^{n}$, i.e., the space of tempered distributions $u$ on $R^{n}$ whose Fourier transforms $\hat{u}$ are measurable functions on $R^{n}$ satisfying

$$
|u|_{s}=(2 \pi)^{-n}\left\{\int\left(1+|\xi|^{2}\right)^{s}|\hat{u}(\xi)|^{2} d \xi\right\}^{1 / 2}<+\infty .
$$

We shall set

$$
H^{-\infty}=\bigcup_{s \in R} H^{s}, \quad H^{\infty}=\bigcap_{s \in R} H^{s}
$$

When $E$ is either $H^{-\infty}$ or $H^{\infty}$, we shall denote by $\Lambda^{P} C^{\infty}(\Omega ; E)$ the sheaf of germs of $C^{\infty} p$-forms in $\Omega$, valued in $E$ and by $\Lambda^{p} \mathscr{D}^{\prime}(\Omega ; E)$ the sheaf of germs of $E$-valued $p$-currents in $\Omega$.

Let $b(t, \xi)=\sum_{j=1}^{\nu} b_{j}(t, \xi) d t_{j}$ be a $C^{\infty}$ one-form in $\Omega$ depending on the parameter $\xi$ of $R_{n}$, the dual of $R^{n}$, where $b_{j}(t, \xi)$ is a pseudodifferential operator from $E$ into $E$. We shall then form a differential-pseudodifferential

Received by the editors November 13, 1977.

AMS (MOS) subject classifications (1970). Primary 35H05.

Key words and phrases. Complex, pseudodifferential operator, hypoellipticity.

(1) American Mathematical Society 1978 
operator

$$
D=d_{t}+b\left(t, D_{x}\right) \Lambda
$$

where $\Lambda$ is the exterior multiplication on $E$. This enables us to define a linear operator

$$
D^{p}: \Lambda^{p} C^{\infty}(\Omega ; E) \rightarrow \Lambda^{p+1} C^{\infty}(\Omega ; E)
$$

for each $p=0,1, \ldots, \nu-1$.

We shall assume that $b(t, \xi)=d_{t} B(t, \xi)$ where $B(t, \xi)$ is real valued and positive-homogeneous of degree one in $\xi$-variable.

Now let us consider the complex of the sheaves

$$
\ldots \Lambda^{p} C^{\infty}\left(\Omega ; H^{-\infty}\right) \stackrel{D^{p}}{\rightarrow} \Lambda^{p+1} C^{\infty}\left(\Omega ; H^{-\infty}\right) \rightarrow \ldots
$$

We note that for any $u \in \Lambda^{p} C^{\infty}\left(\Omega ; H^{-\infty}\right)$ and any $f \in \Lambda^{p+1} C^{\infty}\left(\Omega ; H^{-\infty}\right)$, $D u=f$ and $f \in \Lambda^{p+1} C^{\infty}\left(\Omega ; H^{\infty}\right)$ does not imply, in general, $u \in \Lambda^{p} C^{\infty}(\Omega$; $\left.H^{\infty}\right)$. In fact, there can be an element $v \in \Lambda^{p} C^{\infty}\left(\Omega ; H^{-\infty}\right)$ such that $D v=0$ but $v \notin \Lambda^{p} C^{\infty}\left(\Omega ; H^{\infty}\right)$ (cf. [3, Theorem III.1.1]). Therefore, with more generality, when $p>0$, we think of the following problem; under which conditions, if $D u=f$ and $f \in \Lambda^{p+1} C^{\infty}\left(\Omega ; H^{-\infty}\right)$, there exists $v \in$ $\Lambda^{p-1} C^{\infty}\left(\Omega ; H^{-\infty}\right)$ such that $u-D v \in \Lambda^{p} C^{\infty}\left(\Omega ; H^{\infty}\right)$.

We recall that the space of $p$ th microfunctions are, by definition,

$$
\Lambda^{p} C^{\infty}\left(\Omega ; H^{-\infty}\right) / \Lambda^{p} C^{\infty}\left(\Omega ; H^{\infty}\right) .
$$

Then our complex $\left\{\Lambda^{p} C^{\infty}\left(\Omega ; H^{-\infty}\right) ; D\right\}$ induces in a natural way, a complex of micro-functions, namely,

$$
\begin{aligned}
\ldots & \rightarrow \Lambda^{p} C^{\infty}\left(\Omega ; H^{-\infty}\right) / \Lambda^{p} C^{\infty}\left(\Omega ; H^{\infty}\right) \\
& \stackrel{D^{p}}{\rightarrow} \Lambda^{p+1} C^{\infty}\left(\Omega ; H^{-\infty}\right) / \Lambda^{p+1} C^{\infty}\left(\Omega ; H^{\infty}\right) \rightarrow \ldots
\end{aligned}
$$

Then the previous problem is precisely the problem: when $p>0$, under which conditions $\dot{D} \dot{u}=0$ implies $\dot{u} \in \operatorname{Im} \dot{D}$ for any $p$ th microfunction $\dot{u}$. Under this motivation, we adopt the following definition.

Definition 1.1. $D$ is hypoelliptic in the dimension $p(p=1,2, \ldots, \nu-1)$ if and only if $\operatorname{Im} \dot{D}^{p-1}=\operatorname{ker} \dot{D}^{p}$.

We note that the above definition is a true generalization of the hypoellipticity defined when $p=0$ in [3] if we set $\Lambda^{-1} C^{\infty}(\Omega ; E)=0$ for $E=H^{-\infty}$ or $H^{\infty}$.

The necessary and sufficient conditions for the hypoellipticity in this case are given in [3].

We also remark that, in the above whole arguments, $\Lambda^{p} C^{\infty}\left(\Omega ; H^{-\infty}\right)$ may be replaced by $\Lambda^{p} \mathscr{D}^{\prime}\left(\Omega ; H^{-\infty}\right)$ but keeping $\Lambda^{p} C^{\infty}\left(\Omega ; H^{\infty}\right)$ as the same. However, we would not have any gain in generality by this replacement (cf. [3, Proposition III.2.2]).

2. Sufficient conditions for the hypoellipticity. Let the complex $\left\{\Lambda^{p} C^{\infty}(\Omega\right.$; $\left.H^{-\infty}\right) ; D$ \} be given as in $\S 1$. 
When $U$ is an open subset of $R^{\nu}$, we have $p$ th DeRham homology group $H_{p}(U)$ in $U$ induced by the complex

$$
\ldots \rightarrow \Lambda^{p} C_{c}^{\infty}(U) \stackrel{d}{\rightarrow} \Lambda^{p+1} C_{c}^{\infty}(U) \rightarrow \ldots
$$

with the usual exterior derivative $d$. Let $U$ and $V$ be open subsets of $R^{\nu}$. Let $\theta$, $\theta^{\prime}$ be two open subsets of $U$ such that $\theta^{\prime} \subset \theta \cap V$. For any $\xi \in R_{n}$ and for any real number $\gamma$, we write

$$
\theta(\xi, \gamma)=\{t \in \theta ; B(t, \xi)<\gamma\}
$$

and similarly with $\theta^{\prime}$ substituted for $\theta$. The natural injections of $\theta^{\prime}(\xi, \gamma)$ into $V$ and into $\theta(\xi, \gamma)$, respectively, give rise to two homomorphisms

$$
\begin{aligned}
H_{p}\left(\theta^{\prime}(\xi, \gamma)\right) \stackrel{i_{p}}{\underset{j_{p}}{\longrightarrow}} H_{p}(V) \\
H_{p}(\theta(\xi, \gamma))
\end{aligned}
$$

We introduce a property $\Psi_{p}$ as follows:

$\left(\Psi_{p}\right)$ For every point $t_{0}$ in $\Omega$ and to every open neighborhood $U$ of $t_{0}$, there is another open neighborhood $V$ of $t_{0}, V \subset U$, such that for every open subset $\theta^{\prime} \subset V$, there is a relatively compact open subset $\theta$ of $U$ containing $\theta^{\prime}$ such that for any $\xi \in R^{n}$ and for any real number $\gamma$

$$
\operatorname{Ker} i_{p} \subset \operatorname{Ker} j_{p} \text {. }
$$

THEOREM 2.1. If $\Omega$ has the property $\Psi_{p}$ and $\Psi_{p-1}(p>0)$, then $D$ is hypoelliptic in the dimension $p$.

Proof. We note that our operator $D$ can be extended in a natural way (cf. [3]) as a linear operator

$$
D^{p}: \Lambda^{p} \mathscr{D}^{\prime}\left(\Omega ; \mathrm{FLoc}^{2}\right) \rightarrow \Lambda^{p+1} \mathscr{D}^{\prime}\left(\Omega ; \mathrm{FLoc}^{2}\right)
$$

where FLoc $^{2}$ are the space of generalized functions on $R^{n}$ whose Fourier transforms are locally $L^{2}$-functions on $R_{n}$. Taking Fourier transform we have a linear operator

$$
\left.\left.\hat{D}^{p}: \Lambda^{p} \mathscr{Q}\right)^{\prime}\left(\Omega ; \operatorname{Loc}^{2}\right) \rightarrow \Lambda^{p+1} \mathscr{D}\right)^{\prime}\left(\Omega ; \operatorname{Loc}^{2}\right) .
$$

When $E$ is either $H^{-\infty}$ or $H^{\infty}$, we set $B_{b}^{p+1} C^{\infty}(\Omega ; E)$ the space of elements $f \in \Lambda^{p} C^{\infty}(\Omega ; E)$ such that for almost every $\xi \in R_{n}$, the $p+1$ form $e^{B(t, \xi)} \hat{f}(\xi$, $t)$ is a coboundary in the complex $\left\{\Lambda^{p} \mathscr{D}{ }^{\prime}\left(\Omega ; \operatorname{Loc}^{2}\right) ; \hat{D}^{p}\right\}$.

Now let $f$ be a germ of a $p+1$ form at $t_{0} \in \Omega$ such that $D u=f$ for some $u \in \Lambda^{p}\left(C^{\infty} ; H^{-\infty}\right)$. We may take a neighborhood $U$ of $t_{0}$ to be homologically trivial and may assume $f$ is defined on $U$ as a $p+1$ form. Since $D f=0$, $f$ belongs to $B_{b}^{p+1} C^{\infty}\left(U, H^{\infty}\right)$ since $B_{D}^{p+1} C^{\infty}(U ; E)$ is equal to the kernel of $D: \Lambda^{p} C^{\infty}(U ; E) \rightarrow \Lambda^{p+1} C^{\infty}(U ; E)$ when $E$ is either $H^{-\infty}$ or $H^{\infty}$ and when $U$ is homologically trivial (cf. [3, II.4]). Since $\Omega$ has the property $\Psi_{p}$, we may choose a neighborhood $V$ of $t_{0}$ satisfying the condition $\Psi_{p}$. Then using the [3, Theorem II.1.2], we conclude that, for any relatively compact open subset $W$ 
of $V, D w=f$ has a solution $w \in \Lambda^{p} C^{\infty}\left(W ; H^{\infty}\right)$ in $W$.

Since $D u=f$ and $D w=f$, we have

$$
D(u-w)=f \text { in } W .
$$

Note that $u-w \in \Lambda^{p} C^{\infty}\left(W ; H^{-\infty}\right)$.

We may take $W$ to be homologically trivial so that $u-w \in B_{D}^{p} C^{\infty}(W$; $\left.H^{-\infty}\right)$. Since $\Omega$ has the property $\Psi_{p-1}$, using [3, Theorem II.1.2] again, there exists $v \in \Lambda^{p-1} C^{\infty}\left(W^{\prime} ; H^{-\infty}\right)$ for some neighborhood $W^{\prime}$ of $t_{0}$ such that $D v=u-w$. This shows that $u-D v \in \Lambda^{p} C^{\infty}\left(\Omega ; H^{\infty}\right)$, thus completing our proof.

3. The nondegenerate case. In this section let us assume that $B(t, \xi)$ satisfies that $\forall \xi \in R_{n} \backslash\{0\}$ every critical point of $B(t, \xi)$ in $\Omega$ is nondegenerate, i.e., if $d_{t} B(t, \xi)=0$, then det $\partial_{t}^{2} B(t, \xi) \neq 0$ for any $t \in \Omega$ and $\xi \in R_{n} \backslash\{0\} . \partial_{t}^{2} B(t, \xi)$ stands for the Hessian matrix of $B(t, \xi)$ with respect to $t$. The index of $B\left(t, \xi^{9}\right)$ at $t^{0}$ is, by definition, the number $\mu=\mu\left(t^{0}, \xi^{0}\right)$ of strictly negative eigenvalues of $\partial_{t}^{2} B\left(t^{0}, \xi^{0}\right)$.

DEFINITION 3.1. We say that for $p=1, \ldots, \nu$ the property $Z(p)$ holds for $D$ on $\Omega$ if $\forall \xi \in R_{n} \backslash\{0\}$, the index of $B(t, \xi)$ at every critical point $t$ of $B(\cdot, \xi)$ in $\Omega$ is not equal to $p$.

THEOREM 3.1. Suppose that, for all $\xi \in R_{n} \backslash\{0\}$, every critical point of the function $B(\cdot, \xi)$ in $\Omega$ is nondegenerate. Let $p$ be an integer such that $0<p \leqslant \nu$. Then $D$ is hypoelliptic in the dimension $p$ if $Z_{(p)}$ and $Z_{(p+1)}$ hold for $D$ on $\Omega$.

Proof. Let $f \in \Lambda^{p+1} C^{\infty}\left(\Omega ; H^{\infty}\right)$ such that $D u=f$ for some $u \in \Lambda^{p} C^{\infty}(\Omega$; $\left.H^{-\infty}\right)$. Since $Z_{(p+1)}$ holds for $D$ and $D f=0$, using [3, Theorem II.4.3]. There exists $w \in \Lambda^{p} C^{\infty}\left(\Omega ; H^{\infty}\right)$ such that $D w=f$. Since $D u=f, D(u-w)=0$. Since $Z_{(p)}$ holds for $D$ and $D(u-w)=0$, using [3, Theorem II.4.3] again, we have $v \in \Lambda^{p-1} C^{\infty}\left(\Omega ; H^{-\infty}\right)$ such that $D v=u-w$. Thus $u-D v \in$ $\Lambda^{p} C^{\infty}\left(\Omega ; H^{\infty}\right)$, completing the proof.

COROllary 1. With the same hypothesis as in the theorem, let $E$ be either $H^{-\infty}$ or $H^{\infty}$. If the complex $\left\{\Lambda^{p} C^{\infty}(\Omega ; E) ; D\right\}$ is globally locally solvable in the $p$-and $(p+1)$-dimensional levels, then $D$ is hypoelliptic in the dimension $p$.

Proof. $Z_{(p)}$ (resp. $\left.Z_{(p+1)}\right)$ is equivalent to the global local solvability of the complex $\left\{\Lambda^{p} C^{\infty}(\Omega ; E) ; D\right\}$ at the $p$-dimensional (resp. $(p+1)$-dimensional) level. Thus the previous theorem completes our proof.

COROLlaRy 2. With the same hypothesis as in the theorem, we assume further that $D$ is antipodal, i.e., $B(t,-\xi)=-B(t, \xi)$ for every $\xi \in R_{n} \backslash\{0\}$. Then $D$ is hypoelliptic in the dimension $p$ if $Z_{(p)}, Z_{(\nu-p)}, Z_{(p+1)}$ and $Z_{(\nu-p-1)}$ hold for $D$ on $\Omega$.

Proof. If $D$ is antipodal, then the symmetry $\xi \rightarrow-\xi$ changes the index $\mu$ of $B(t, \xi)$ of an arbitrary critical point of $B(\cdot, \xi)$ into $\nu-\mu$. Thus applying the previous theorem completes the proof. 
We note that the $\nu$-dimensional induced boundary complex introduced in [2] can be transformed by the Fourier integral operator modulo smooth operators to the $D$ complex on $R^{\nu}$ with $n=\nu+1$ and $b_{j}(t, \xi)= \pm t_{j}|\xi|$ (cf. [1]). Since the boundary operator $\partial_{b}$ is antipodal, we have the following corollary.

Corollary 3. Let

$$
\ldots \rightarrow B^{0, p} \stackrel{\partial_{b}}{\rightarrow} B^{0, p+1} \rightarrow \ldots
$$

be the $\nu$-dimensional induced boundary complex. Then $\partial_{b}$ is hypoelliptic in the dimension $p>0$ in the sense analogous to Definition 1.1 if the Levi matrix at the critical point of $\partial_{b}$ has no index identical to $p, \nu-p, p+1, \nu-p-1$.

Proof. Since the index of the Levi matrix of $\partial_{b}$ at $(t, \xi)$ is the same as the index of the Hessian matrix at $(t, \xi)$, the corollary follows from the previous theorem and [3, Proposition III.2.2] in p-dimensional form.

\section{REFERENCES}

1. L. Boutet de Monvel, Hypoelliptic operators with double characteristics and related pseudodifferential operators, Comm. Pure Appl. Math. 27 (1974), 589-639.

2. G. B. Folland and J. J. Kohn, The Neumann problem for the Cauchy-Riemann complex, Ann. of Math. Studies, Princeton Univ. Press, Princeton, N. J., 1972.

3. F. Treves, Study of a model in the theory of complexes of pseudodifferential operators, Ann. of Math. (2) 104 (1976), 269-324.

Department of Mathematics, Seoul National University, Seoul, Korea 